\title{
Above-Knee Amputation
}

National Cancer Institute

\section{Source}

National Cancer Institute. Above-Knee Amputation. NCI Thesaurus. Code C51772.

Surgical removal of all or part of the leg, made at any point of the femur. 